\title{
Mechanism of trans-translation revealed by in vitro studies
}

\author{
Hyouta Himeno ${ }^{1,2}$ *, Daisuke Kurita ${ }^{1,2}$ and Akira Muto \\ ${ }^{1}$ Department of Biochemistry and Molecular Biology, Faculty of Agriculture and Life Science, Hirosaki University, Hirosaki, Japan \\ ${ }^{2}$ RNA Research Center, Hirosaki University, Hirosaki, Japan
}

\section{Edited by: \\ Kenneth C. Keiler, Pennsylvania State University, USA}

\section{Reviewed by:}

Paras Jain, Albert Einstein College of Medicine, USA

Nobukazu Nameki, Gunma University, Japan

\section{${ }^{*}$ Correspondence:}

Hyouta Himeno, Department of Biochemistry and Molecular Biology, Faculty of Agriculture and Life Science, Hirosaki University, Hirosaki 036-8561, Japan

e-mail:himeno@cc.hirosaki-u.ac.jp
tmRNA is a bacterial small RNA having a structure resembling the upper half of tRNA and its $3^{\prime}$ end accepts alanine followed by binding to EF-Tu like tRNA. Instead of lacking a lower half of the cloverleaf structure including the anticodon, tmRNA has a short coding sequence for tag-peptide that serves as a target of cellular proteases. An elaborate coordination of two functions as tRNA and mRNA facilitates an irregular translation termed trans-translation: a single polypeptide is synthesized from two mRNA molecules. It allows resumption of translation stalled on a truncated mRNA, producing a chimeric polypeptide comprising the C-terminally truncated polypeptide derived from truncated mRNA and the C-terminal tagpeptide encoded by tmRNA. Trans-translation promotes recycling of the stalled ribosomes in the cell, and the resulting C-terminally tagged polypeptide is preferentially degraded by cellular proteases. Biochemical studies using in vitro trans-translation systems together with structural studies have unveiled the molecular mechanism of trans-translation, during which the upper and lower halves of tRNA are mimicked by the tRNA-like structure of tmRNA and a tmRNA-specific binding protein called SmpB, respectively. They mimic not only the tRNA structure but also its behavior perhaps at every step of the trans-translation process in the ribosome. Furthermore, the $\mathrm{C}$-terminal tail of $\mathrm{SmpB}$, which is unstructured in solution, occupies the mRNA path in the ribosome to play a crucial role in trans-translation, addressing how tmRNA.SmpB recognizes the ribosome stalled on a truncated mRNA.

Keywords: trans-translation, tmRNA, SmpB, ribosome rescue system, molecular mimicry

\section{INTRODUCTION}

As mRNA acts as a messenger of the genetic information encoded by the genome, while tRNA acts as a tool for decoding, it is reasonable that they are separated molecules. However, both features are equipped with a single small RNA molecule called tmRNA (also known as 10Sa RNA or SsrA). The presence of the tRNA-like secondary structure with several tRNA-specific sequences including the $3^{\prime}$-terminal CCA sequence in tmRNA was first found in 1994 (Komine et al., 1994; Ushida et al., 1994; Figure 1A), and thereafter several other structural and functional similarities to tRNA, such as the aminoacylation capacity with alanine (Komine et al., 1994; Ushida et al., 1994), the binding capacity to the ribosome (Ushida etal., 1994; Tadaki et al., 1996), the $5^{\prime}$ processing by RNase $P$ (Komine et al., 1994) and the binding capacity to EF-Tu after aminoacylation (RudingerThirion et al., 1999; Barends et al., 2000, 2001; Hanawa-Suetsugu et al., 2001) and the presence of tRNA-specific base modifications (Felden et al., 1998), have been reported, although the anticodon has never been found in tmRNA, a few hundred nucleotides in length. The function as mRNA has been suggested by an observation that a peptide of 10 -amino acid sequence encoded by tmRNA is attached to the truncated C-termini of polypeptides that are exogenously expressed in Escherichia coli with an alanine residue of unknown origin in between them (Tu et al., 1995; Keiler et al., 1996; Figure 1B). Thus the trans-translation model has been proposed: Ala-tmRNA receives the nascent polypeptide from peptidyl-tRNA stalled on a truncated mRNA to continue translation by exchanging the template from truncated mRNA to the tag-encoding region on tmRNA (Keiler et al., 1996). It can address the missing origin of the first alanine residue of the tag-peptide, which is derived from the alanine moiety aminoacylated to tmRNA. This model has been supported by an in vitro study showing that the tmRNA-encoded tag-peptide is synthesized using E. coli cell extract depending on the presence of poly $(\mathrm{U})$ as a truncated mRNA and on the aminoacylation capacity of tmRNA (Muto et al., 1996; Himeno et al., 1997; Nameki et al., 1999a). The C-terminal four amino acid sequence of the tag-peptide, ALAA, serves as a target for a periplasmic protease, Tsp (Keiler etal., 1996), and cytoplasmic ATP-dependent proteases (AAA+ proteases) including ClpXP, ClpAP, FtsH, and Lon (Gottesman etal., 1998; Herman etal., 1998; Flynn et al., 2001; Choy et al., 2007). In fact, trans-translation products, namely, tagged polypeptides, are hardly detectable but become accumulated in cell when the terminal ALAA sequence of the tag-peptide is engineered (Roche and Sauer, 2001; Collier et al., 2002; Fujihara et al., 2002). tmRNA is perhaps absolutely distributed among bacteria and it has also been found in chloroplasts or mitochondria of some eukaryotes (Gueneau de Novoa and Williams, 2004; Jacob et al., 2004), but not in the cytoplasm of eukaryotes or archaebacteria. Taken together, trans-translation has been considered as the bacterial system that facilitates recycling of the ribosome stalled on a truncated mRNA lacking a stop codon and to prevent the premature polypeptide from accumulation by adding a tag to its C-terminus for degradation (Keiler et al., 1996; Muto et al., 1998) as well as to promote decay of causative truncated mRNA (Yamamoto et al., 2003). In addition, trans-translation 
A

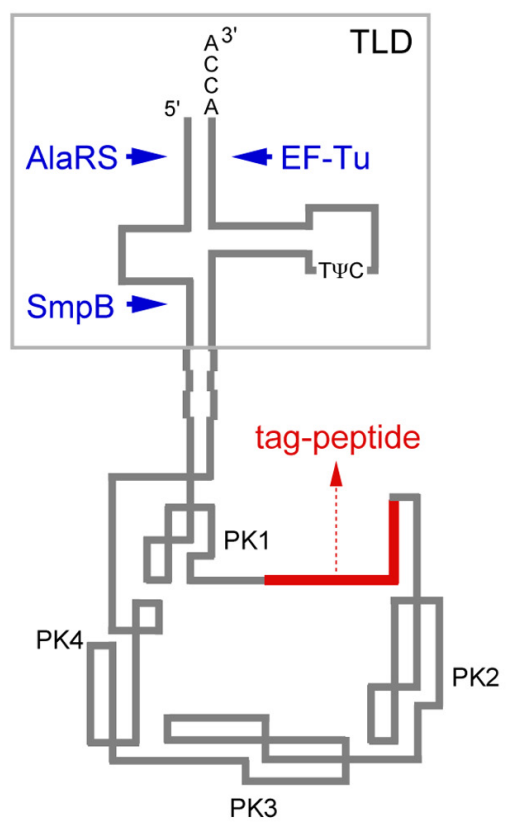

FIGURE 1 | tmRNA and trans-translation. (A) Secondary structure model of tmRNA. The $3^{\prime}$-end CCA sequence, amino acid acceptor stem, D-arm and T-arm with two typical tRNA-specific modified nucleotides, 5-methyluridine (T) and pseudouridine ( $\psi$; Felden et al., 1998), are present in TLD of E. coli tmRNA to which alanyl-tRNA synthetase (AlaRS), EF-Tu and SmpB bind. The tag-encoding sequence (red) is surrounded by four pseudoknot structures
B

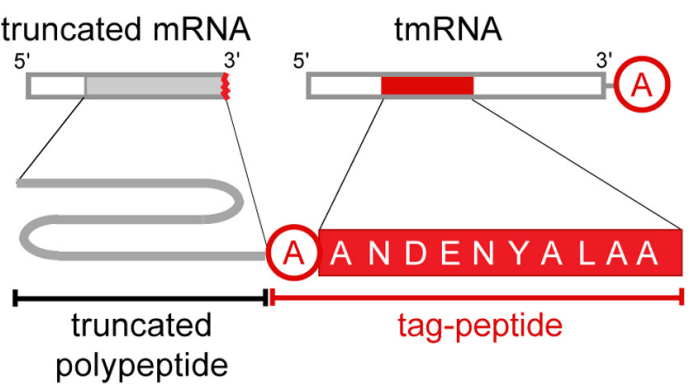

has been shown to participate in a wide variety of cellular events (Keiler, 2008), e.g., cell viability (Hutchison et al., 1999; Huang et al., 2000; Akerley et al., 2002; Ramadoss et al., 2013a), stress response (Muto et al., 2000; Ranquet and Gottesman, 2007; Shin and Price, 2007), cell cycle (Keiler and Shapiro, 2003a,b) and regulation of gene expression (Abo et al., 2000; Abe etal., 2008; Ujiie etal., 2009; Chadani etal., 2011a; Garza-Sánchez et al., 2011).

tmRNA is about 4- or 5-fold larger than tRNA, and the central $4 / 5$, which does not participate in the tRNA-like structure, have a unique pseudoknot-rich secondary structure forming a large ring that surrounds the coding region for tag-peptide (Figure 1A; Felden etal., 1997; Nameki etal., 1999b). As several aspects of this novel system became clearer, new questions have arisen. How does tmRNA select the stalled ribosome? How does tmRNA enter the A-site of the ribosome without an anticodon? How does the tag-encoding region of tmRNA substitute for truncated mRNA? How does tmRNA about 5-fold larger than tRNA move in the narrow space of the ribosome to continue translation? How is the resuming point on tmRNA determined? The trans-translation reaction can proceed in vitro using isolated components from $E$. coli (Shimizu and Ueda, 2002; Ivanova et al., 2004; Konno et al., 2007) or Thermus thermophilus (Takada et al., 2007), revealing that a stalled ribosome with a truncated mRNA and a peptidyl-tRNA, Ala-tmRNA, elongation factors and a tmRNA-binding protein called SmpB are the minimal requirement for the first few steps of trans-translation including the initial binding of tmRNA to
(PK1-PK4). (B) Schematic representation of trans-translation. The tag-peptide sequence varies depending on the bacterial species, and that of $E$. coli is presented. A short peptide encoded by tmRNA is attached to the truncated C-terminus of a polypeptide from truncated mRNA with an alanine residue (circled in red) that is derived from the alanine moiety aminoacylated to tmRNA in between them. the stalled ribosome, peptidyl-transfer from peptidyl-tRNA to Ala-tmRNA and decoding of the first codon on tmRNA (Kurita et al., 2012). Another tmRNA-binding protein, a ribosomal protein S1 has also been identified (Wower et al., 2000). It is dispensable for trans-translation at least until the first peptidyltransfer reaction (Qi et al., 2007; Takada et al., 2007), although it might have a role in a later process of trans-translation (Saguy et al., 2007; Shi et al., 2011). S1 is absent in a group of Gram-positive bacteria, although tmRNA still acts in Bacillus subtilis belonging to this group (Muto et al., 2000; Ito et al., 2002).

It has recently been revealed that bacterial cells have a variety of systems to rescue the stalled ribosome. Since it is universally conserved in bacteria, trans-translation is considered as the primary ribosome rescue system in the bacterial cells. Besides, the trans-translation system has distinct features including employment of a small RNA as the main player and addition of a tag for degradation to the nascent polypeptide. In this review, we focused on the molecular mechanism of the apparently spectacular reaction of trans-translation involving co-translational mRNA switching.

\section{SmpB}

SmpB is essential for trans-translation in vivo (Karzai et al., 1999) and in vitro (Hanawa-Suetsugu et al., 2002; Shimizu and Ueda, 2002). SmpB has a globular core with a C-terminal tail unstructured in solution (Dong et al., 2002; Someya et al., 2003), and the globular domain binds to the tRNA-like domain (TLD) of tmRNA 
to prevent tmRNA from degradation and enhance aminoacylation of tmRNA (Figure 1A; Barends et al., 2001; Hanawa-Suetsugu et al., 2002; Shimizu and Ueda, 2002; Nameki et al., 2005). A crystal structure of a complex of SmpB and a model RNA fragment corresponding to TLD has revealed that the tertiary structure of TLD is indeed similar to the upper half of the L-form structure of the tRNA molecule as has been expected from the tRNA-like primary and secondary structures including the $3^{\prime}$-terminal CCA sequence, the amino acid acceptor stem and T-arm. Intriguingly, the globular domain of SmpB binds to TLD so that it compensates for the lack of the lower half of the L-form structure of tRNA in tmRNA (Figure 2; Gutmann et al., 2003; Bessho et al., 2007). When TLD is put on the upper half of the L-form structure of tRNA, the globular domain of SmpB is superimposed on the anticodon arm, indicating that TLD with the globular domain of SmpB structurally mimics a whole tRNA molecule. Functional mimicry of tRNA by TLD.SmpB has been suggested by a directed hydroxyl radical probing study showing that at least two SmpB molecules have capacity to bind an E. coli $70 \mathrm{~S}$ ribosome, one to the A-site and the other to the P-site, both of which can be superimposed on the lower half of the tRNA molecules in the elongating ribosome (Kurita et al., 2007). An additional mimicry of the upper half of tRNA by TLD would facilitate full mimicry of each tRNA molecule in the elongating ribosome. SmpB in complex with a TLD fragment facilitates polyalanine synthesis in vitro without template mRNA, functionally supporting the molecular mimicry of tRNA and mRNA by TLD.SmpB (Shimizu and Ueda, 2006).

The truncation or mutation of the C-terminal tail of SmpB seriously affects trans-translation in vivo and in vitro, indicating its functional significance (Jacob et al., 2005; Sundermeier et al., 2005; Konno et al., 2007; Kurita et al., 2007, 2010). However, cryoEM studies have failed to identify the C-terminal tail due to lack of resolution (Gillet et al., 2006; Kaur et al., 2006). Its location in the ribosome has been revealed by a directed hydroxyl radical probing study (Kurita et al., 2007). The C-terminal tail of A-site SmpB extends to the downstream tunnel along the mRNA path.
Probing signals from the C-terminal tail of A-site SmpB extends to the downstream tunnel along the mRNA path, and those from its latter half appear at interval of three residues suggesting an $\alpha$-helical structure. In contrast, probing signals from P-site SmpB are localized in a limited area, and thus it is likely to be in a folded conformation around the mRNA path in the P-site. Thus three different modes of conformations of the C-terminal tail of SmpB have been assumed: an unstructured structure in solution, an extended structure from the A-site to the mRNA entry channel and a folded structure in the P-site (Kurita et al., 2007).

\section{CURRENT MODEL OF TRANS-TRANSLATION}

Based on the structural resemblance of the complex of SmpB and TLD to a tRNA molecule, the binding sites of SmpB shared by translating tRNA, and the interaction of the C-terminal tail of SmpB with the mRNA path, a model of the trans-translation process has been proposed (Figure 3; Kurita et al., 2007, 2010). In this model, TLD.SmpB is assumed to functionally mimic the dynamic behavior of tRNA in the elongating ribosome through all the classical and hybrid states, A/T, A/A, A/P, P/P and P/E states.

After aminoacylation with alanine, tmRNA.SmpB binds to EF-Tu.GTP to make a quaternary complex, and thereafter it enters the vacant A-site of the stalled ribosome. Subsequent GTP hydrolysis of EF-Tu may induce conformational changes of the quaternary complex and the ribosome so that EF-Tu.GDP is released and AlaTLD.SmpB is accommodated in the A-site. During this process, the C-terminal tail of SmpB interacts with the mRNA path downstream from the A-site with an extended structure. This interaction would be successful only when the downstream mRNA is absent. Then Ala-TLD in the A-site receives the nascent polypeptide chain from peptidyl-tRNA in the P-site, and the resulting peptidyl-AlaTLD.SmpB may translocate from the A-site to the P-site. During this process, the C-terminal tail of $\mathrm{SmpB}$ dissociates from the mRNA path and binds to the region around the P-site codon with changing its conformation from the extended structure to the folded structure, which releases mRNA from the ribosome.

\section{A}

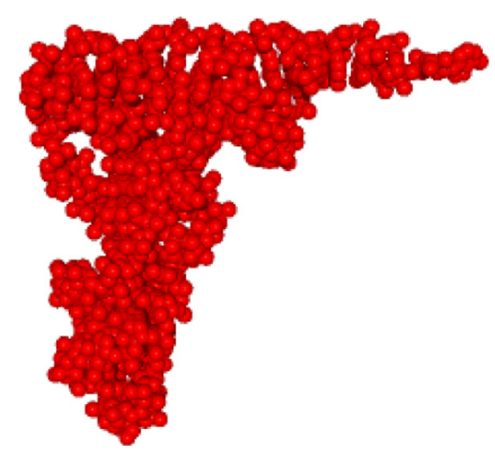

FIGURE 2 | Molecular mimicry of tRNA by the globular domain of SmpB and TLD. (A) Saccharomyces cerevisiae tRNA ${ }^{\text {Phe }}$ represented by space-filling model (PDB ID: 1EHZ). (B) A complex of TLD (the $5^{\prime} 25$ residues and the $3^{\prime} 34$ residues connected by a UUCG loop) of $T$. thermophilus tmRNA represented by space-filling model and the globular
B

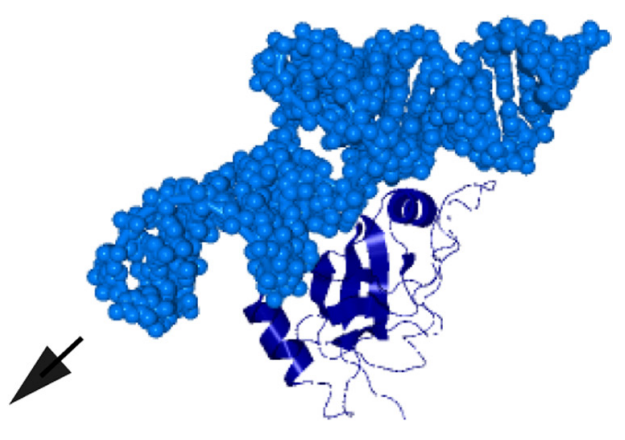

domain (N-terminal 123 of total 144 residues) of SmpB shown in ribbon representation (PDB ID: 2CZJ; Bessho etal., 2007). The remaining region of tmRNA (positions 26-312) is protruded from TLD in a direction similar to that of the long variable arm of class II tRNA, designated by an arrow. 
elongation

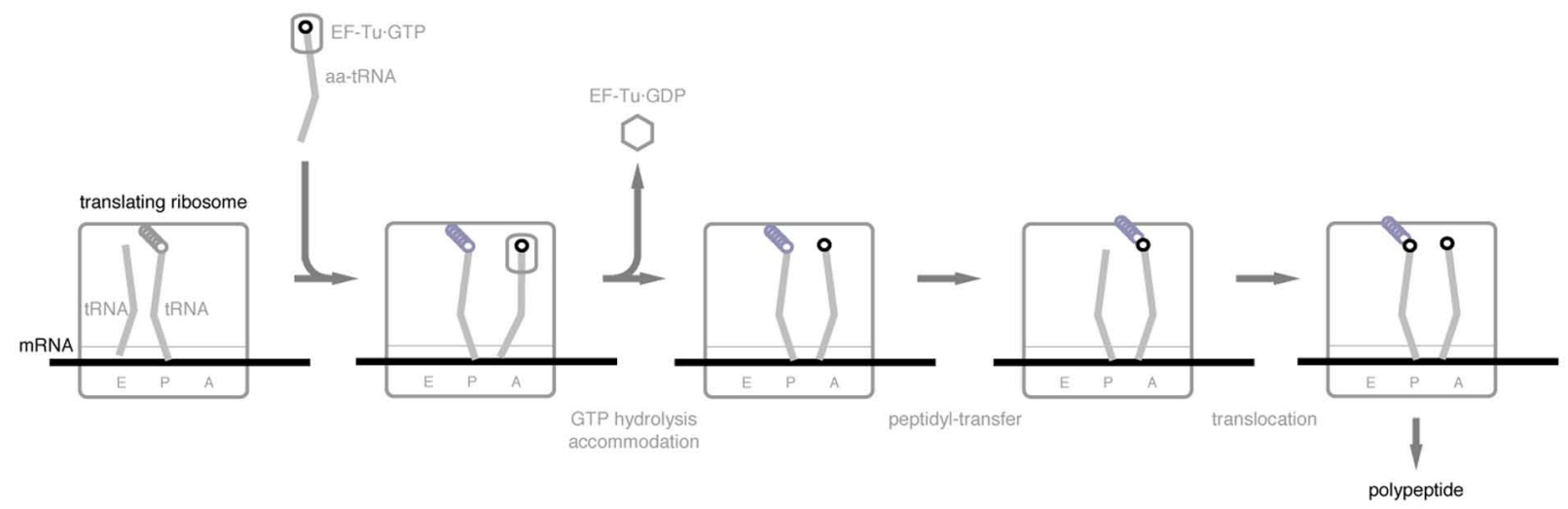

trans-translation



FIGURE 3 | A current model of trans-translation process in comparison with the elongation process of canonical translation. After GTP hydrolysis by EF-Tu, the C-terminal tail of SmpB becomes located along the mRNA path to recognize the stalled ribosome free of mRNA. Upon translocation of peptidyl-Ala-tmRNA.SmpB from the A-site to the P-site, the C-terminal tail undergoes a drastic conformational change from the extended to the folded structures to accommodate the resume codon of tmRNA into the decoding center. During these processes, TLD and the globular domain of SmpB mimic the upper and lower halves of tRNA, respectively.
Just after the movement of peptidyl-Ala-TLD-SmpB to the P-site, the resume codon of tmRNA is positioned at the decoding region.

This model might involve several aspects of the tRNA and mRNA mimicries (1) as the structural unit for entrance to the ribosome with EF-Tu.GTP, (2) as the structural unit for accommodation in the A-site after GTP hydrolysis by EF-Tu, (3) as the structural unit from the A-site to the P-site during translocation, (4) as the mRNA for the first alanine residue of the tag-peptide, and (5) to find the target ribosome in which the downstream mRNA is absent. Although several proteins have been proposed to mimic tRNA such as EF-G (Nissen et al., 1995), SmpB is a special case in that it is assumed to mimic translating tRNA at all the classical and possibly hybrid states from the A-site to the E-site.

\section{TLD.SmpB IN SOME STATES OF TRANS-TRANSLATION COMPLEXES}

The above model is consistent with several structural studies. Cryo-EM maps corresponding to four different states (pre-accommodation, accommodation, translocational intermediate, and post-translocated states) during the trans-translation process have been revealed, where SmpB and TLD occupy the lower and upper halves, respectively, of the A-site or P-site (Kaur et al., 2006; Cheng et al., 2010; Fu et al., 2010; Weis et al., 2010a,b; Ramrath et al., 2012). In addition to the tRNA mimicry by SmpB and TLD, an interaction of the C-terminal tail of SmpB with the downstream mRNA path from the decoding region has explicitly been shown in a crystal structure of T. thermophilus ribosome in complex with a tmRNA fragment, SmpB, EF-Tu.GDP and kirromycin, which mimics the pre-accommodation state of trans-translation (Neubauer et al., 2012). Note that kirromycin is effective for some tmRNA fragments such as TLD but much less effective for full-length tmRNA (Shimizu and Ueda, 2006), and thus the kirromycin complex is not likely to perfectly mimic the pre-accommodation state of trans-translation. In this crystal structure, the C-terminal tail (residues 122-144) extends to the downstream tunnel along the mRNA path with its latter 
half (residues 132-144) forming an $\alpha$-helix as has been suggested by a directed hydroxy radical probing study (Kurita et al., 2007). Some but not all preparations of pre-accommodation state complex contain an additional SmpB molecule near the GTPase associated center of the 50S subunit (Kaur et al., 2006; Weis et al., 2010a), although the relevance of the second SmpB molecule is still controversial (Bugaeva et al., 2008; Felden and Gillet, 2011).

\section{EARLY STEPS OF TRANS-TRANSLATION}

In the elongation process of the canonical translation, a ternary complex (aminoacyl-tRNA.EF-Tu.GTP) enters the elongating ribosome, and only when the anticodon of aminoacyl-tRNA successfully interacts with the codon, GTP is hydrolyzed by EF-Tu to release EF-Tu-GDP from aminoacyl-tRNA and the ribosome. During this process, the codon-anticodon interaction induces conformational change in the decoding center nucleotides, A1492, A1493, and G530 of $16 \mathrm{~S}$ rRNA, leading to domain closure of the 30 S subunit to trigger GTP hydrolysis. However, an alternative interaction should be assumed in the case of trans-translation, since it lacks a codon-anticodon interaction. Direct interaction of E. coli SmpB with the decoding region has been suggested by chemical modification in combination with NMR (NoninLecomte et al., 2009). In a crystal structure of a T. thermophilus pre-accommodation state complex, A1492 and A1493 are in close proximity to the globular domain of SmpB, and G530 stacks with Y126 in the C-terminal tail of SmpB (Figure 4), while the ribosomal protein S12, which is deeply involved in decoding fidelity, has no contact with SmpB (Neubauer et al.,
2012). Consistently, in vitro trans-translation activity is significantly affected by aminoglycosides that flips A1492 and A1493 out of helix 44, while streptomycin, which binds between the juxtaposed residues (C1490 and G1491) and S12, has only a small effect (Takahashi et al., 2003; Konno et al., 2004). Recently, Miller and Buskirk (2014) have found that a mutation in H136 in the C-terminal tail of E. coli SmpB causes serious decrease in GTP hydrolysis. The corresponding residue of $T$. thermophilus SmpB, Y126, is involved in stacking with G530 in a crystal structure of a pre-accommodation state complex of trans-translation (Neubauer etal., 2012). This may highlight the importance of stacking of H136 with G530 for GTP hydrolysis, although any of A1492, A1493 and G530 in E. coli 16S rRNA can be changeable without loss of GTP hydrolytic activity as well as peptidyl-transferase activity of trans-translation (Miller et al., 2011).

Trans-translation is thought to preferentially target the ribosome stalled on a $3^{\prime}$ truncated mRNA, and this situation can be produced by cleavage of intact mRNA around the decoding region. In fact, A-site codon-specific cleavage by RelE (Pedersen et al., 2003) or other unknown endoribonuclease with the help of a $3^{\prime}$ to $5^{\prime}$ exonuclease, RNase II (Garza-Sánchez et al., 2009), has been reported. Besides RelE, several kinds of ribosome-dependent endoribonucleases have been identified in E. coli (Feng et al., 2013). The idea that cleavage of mRNA in the stalled ribosome is the prerequisite for trans-translation has also been supported by in vitro studies: the efficiency of in vitro trans-translation decreases with the increasing length of the $3^{\prime}$ extension of mRNA from the decoding region (Ivanova et al.,
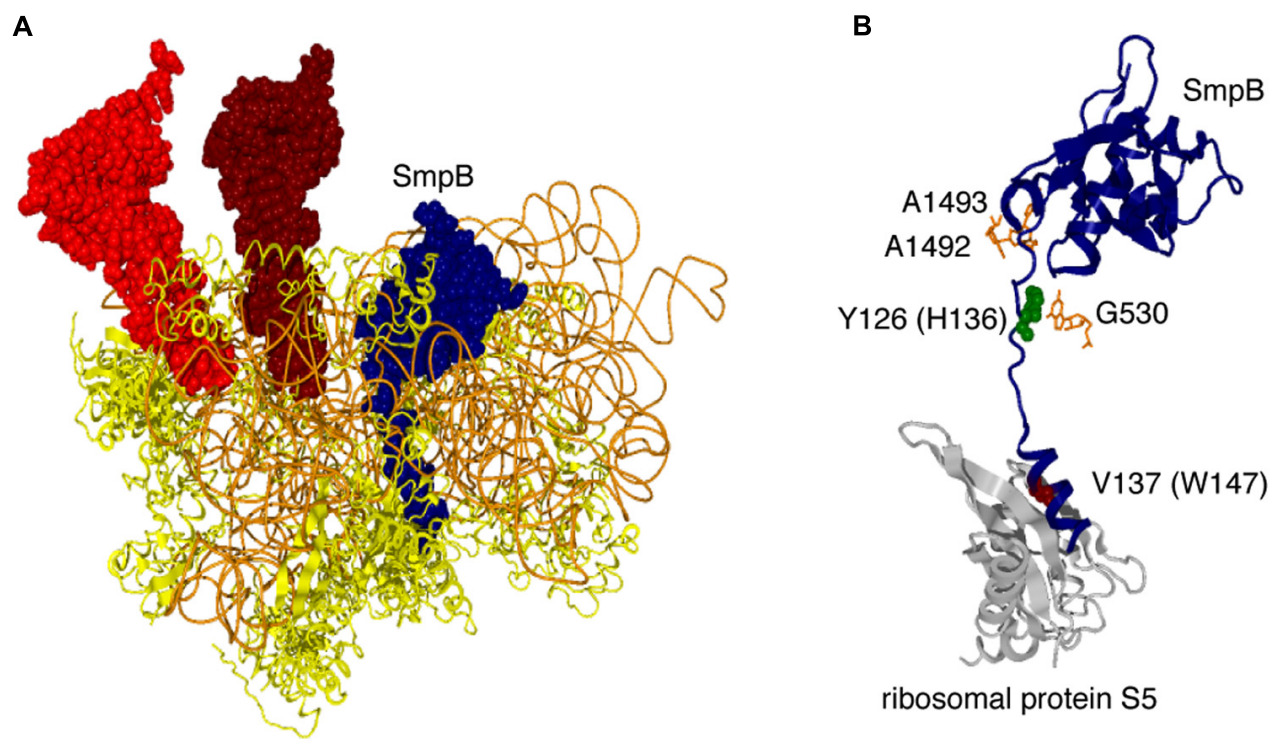

FIGURE 4 | Location of SmpB in a pre-accommodation state complex of trans-translation. (A) SmpB (dark blue, space-filling model), P-site tRNA (brown, space-filling model), E-site RNA (red, space-filling model), 16S rRNA (light brown, wire model), and the ribosomal proteins (yellow, ribbon model) in a crystal structure of $T$. thermophilus ribosome in complex with a tmRNA fragment, SmpB, EF-Tu.GDP and kirromycin (PDB ID: 4ABR), which mimics the pre-accommodation state of trans-translation (Neubauer et al., 2012), are depicted, although the 50S subunit, tmRNA fragment, EF-Tu.GDP and kirromycin are removed. (B) SmpB (dark blue, space-filling model), decoding nucleotides (orange, stick model) and the ribosomal protein S5 (gray, ribbon model) are extracted from the same complex. Y126 (green, space-filling model) and V137 (brown, space-filling model) in the C-terminal tail of SmpB are highlighted. The corresponding residues in E. coli SmpB are shown in parentheses. 
2004; Asano etal., 2005). This can be accounted for by the competition of the $3^{\prime}$-extension of mRNA, if it is longer, with the C-terminal tail of $\mathrm{SmpB}$ for the downstream mRNA tunnel. Consistently, the C-terminal tail of T. thermophilus SmpB in a crystal structure of a pre-accommodation state complex of trans-translation is incompatible with mRNA within a narrow mRNA tunnel (Neubauer et al., 2012). Systematic amino acid substitutions of the C-terminal residues of E. coli SmpB have indicated the significance of a tryptophan residue at 147 (W147) in the middle of the C-terminal tail for binding to the mRNA path required for peptidyl-transfer (Figure 4; Kurita et al., 2007, 2010). The corresponding residue of T. thermophilus SmpB, V137, is involved in hydrophobic interaction with a ribosomal protein S5 in a crystal structure of a pre-accommodation state complex of trans-translation (Neubauer et al., 2012). W147 is changeable without significant loss of GTP hydrolysis, suggesting that the interaction of this residue with the mRNA path occurs after GTP hydrolysis (Kurita et al., 2010). Recently, we found that the efficiency of GTP hydrolysis by EF-Tu reflecting that of the entrance of the Ala-tmRNA.SmpB.EF-Tu.GTP complex to the stalled ribosome is comparable regardless of the length of the $3^{\prime}$-extension of mRNA, and that accommodation or rejection of Ala-tmRNA.SmpB from the ribosome stalled on mRNA is determined after GTP hydrolysis depending on the length of the 3 -extension of mRNA (Kurita et al., manuscript in preparation). It is surprising that Ala-tmRNA.SmpB discriminates the target ribosome in a GTP-wasting manner.

EF-Tu.GTP has a role in the proofreading step in which nearcognate aminoacyl-tRNAs that are escaped from the first selection in the initial binding of the ternary complex before GTP hydrolysis are subjected to the second selection after GTP hydrolysis via recognition of the correct codon-anticodon interaction by the decoding region. Unlike tRNAs, tmRNA is homogeneous in a cell, and thus neither codon-anticodon interaction nor proofreading should be required for trans-translation. GTP hydrolysis would be required mainly for release of EF-Tu, which guarantees the aminoacylation state of tmRNA.

\section{FROM THE A-SITE TO THE P-SITE}

In the canonical elongation process, peptidyl-tRNA translocates with mRNA from the A-site to the P-site. In trans-translation, peptidyl-Ala-tmRNA.SmpB mimicking peptidyl-tRNA, but without mRNA, translocates from the A-site to the P-site. As a consequence, deacylated tRNA and truncated mRNA are pushed from the P-site to the E-site presumably by the globular domain and the folded C-terminal tail of $\mathrm{SmpB}$, respectively, facilitating their dissociations from the ribosome. As in the canonical translation, this process might be driven by EF-G. GTP. In fact, EF$G$ stimulates release of deacylated tRNA and truncated mRNA (Ivanova etal., 2005). Concomitantly, the resume codon on tmRNA for the tag-peptide should be set at the decoding region. A folded rather than extended conformation of the C-terminal tail of SmpB around the P-site as observed in a directed hydroxyl radical probing study might be preferable for both pushing mRNA out of the P-site and setting the resume codon on tmRNA in the A-site (Kurita et al., 2007). Translocation usually involves the ratchet-like rotation between the two ribosomal subunits (Frank and Agrawal, 2000). Comparison of the cryo-EM structures of the accommodation and post-translocated state complexes have suggested that the ribosome in which peptidyl-Ala-tmRNA.SmpB occupies the A-site also undergoes a ratchet-like rotation upon translocation to induce a significant conformational change of a bridge between the $30 \mathrm{~S}$ and $50 \mathrm{~S}$ subunits (bridge B1a), which serves as the barrier between the A-site and P-site, thereby allowing peptidyl-Ala-TLD.SmpB to move from the A-site to the P-site (Weis et al., 2010b; Felden and Gillet, 2011). Consistently, bridge $\mathrm{Bla}$ is open in another cryo-EM map of a translocational intermediate complex containing EF-G and fusidic acid (Ramrath et al., 2012). In this complex, latch, which is usually closed by the interaction between the head (helix 34) and body (the G530 region) to form the mRNA tunnel, is open to introduce the coding region of tmRNA into the decoding center (Ramrath et al., 2012). Meanwhile, the large ring comprised of the central four pseudoknot structures (PK1-PK4) of tmRNA (Felden et al., 1997; Nameki et al., 1999b) keeps surrounding the beak of the $30 \mathrm{~S}$ subunit through the pre-accommodation to the posttranslocated states (Fu et al., 2010; Weis et al., 2010b; Ramrath et al., 2012).

\section{DETERMINATION OF THE RESUME CODON}

The coding region for the tag-peptide starts from the position about 10 nucleotides downstream of PK1. Then a question arises as to what determines the first (resume) codon for tag-peptide. It seems reasonable to assume that some sequence or structural unit on tmRNA is fixed somewhere on the ribosome to set the first codon on the decoding center just after translocation. Changing the span between PK1 and the coding region does not affect the resume codon selection, indicating the lack of significance of PK1 for determination of the initiation point (Lee et al., 2001). In vivo and in vitro studies have revealed the significance of the sequence between PK1 and the tag-encoding region, especially at positions -6 to +1 (Williams et al., 1999; Lee et al., 2001). In vitro trans-translation system can identify not only the efficiency of the first peptidyl-transfer of trans-translation but also the second amino acid of the tag-peptide, which enables us to specify the start point of the tag-translation (Lee et al., 2001; Konno et al., 2007; Takada et al., 2007; Kurita et al., 2011). Using this system, we have found that some point mutations within the span of -6 to +1 reduce the trans-translation efficiency and/or shift the resuming point of translation on tmRNA by -1 or +1 , indicating that this region serves not only as the enhancer of trans-translation but also as the determinant for the resume codon for the tag-peptide (Lee et al., 2001; Konno et al., 2007).

Then the next question arises as to what recognizes this sequence. It has been shown that $U$ at position -5 is protected from chemical modification by the globular domain of $E$. coli SmpB (Konno et al., 2007). This protection is suppressed by a point mutation in the TLD required for SmpB binding, indicating that a single SmpB molecule bridges two separated domains of tmRNA. The interaction of $S m p B$ with $U$ at -5 would make more sense upon selection of the resume codon for tag-peptide translation in the ribosome. Mutations that induce -1 and +1 shifts of the start point of tag-peptide translation in vitro also 
shift the site of protection at position -5 from chemical modification by -1 and +1 , respectively (Konno et al., 2007), indicating the significance of the fixed span between the site of interaction on tmRNA with SmpB and the start point of tag-peptide translation. Thus the interaction between tmRNA and SmpB would be more important for resume point determination than that between tmRNA and the ribosome. A functional interaction of the upstream region in tmRNA with $\mathrm{SmpB}$ has been supported by a genetic study showing that a mutation at position -4 of E. coli tmRNA that inactivates trans-translation is suppressed by some mutations in SmpB (Watts et al., 2009). It is also consistent with current cryo-EM studies suggesting that the nucleotides at -4 and -5 are in close contact with $\mathrm{SmpB}$ around the P-site in the post-translocated (resume) state of trans-translation (Fu et al., 2010; Weis et al., 2010b). Another in vivo study has suggested the importance of the C-terminal tail of SmpB and its interaction with the start GCA codon on tmRNA for determination of the start point of tag-peptide translation (Camenares et al., 2013). This again emphasizes the importance of the interaction between tmRNA and SmpB.

Shift of the start point of tag-peptide translation can also be induced by some kinds of aminoglycosides (Takahashi et al., 2003; Konno et al., 2004), which cause miscoding of translation by binding to A1492 and A1493 to modulate the conformation of the decoding region (A-site).

\section{DIVERSITY OF RESCUE SYSTEMS OF THE STALLED RIBOSOME}

Peptidyl-tRNA often drops-off from the ribosome stalled within the first few rounds of elongation, followed by hydrolysis of detached peptidyl-tRNA by Pth (peptidyl-tRNA hydrolases; Heurgué-Hamard et al., 1998; Gong et al., 2007; Singh et al., 2008). Drop-off of peptidyl-tRNA can also occur at the $3^{\prime}$ end of mRNA (Kuroha et al., 2009) and the temperature-sensitive phenotype of Pth is suppressed by overexpression of tmRNA (Singh and Varshney, 2004). In 2010s, we came to know that the rescue systems of the bacterial stalled ribosome are more diversified than we have imagined (Figure 5). For example, EF4 (also known as LepA), which is usually sequestered in the cell membrane, is released into cytoplasm to rescue the translational arrest by back translocation upon high intracellular magnesium ion concentration or low temperature (Pech et al., 2011). The ribosome often stalls at the Pro-Pro-Pro or Gly-Pro-Pro arrest sequence, and EF-P can alleviate this arrest by modulating the peptidyl-transferase center (Doerfel et al., 2013; Ude et al., 2013). Unlike the drop-off/Pth system, EF-4 and EF-P promote resumption of elongation process to complete translation rather than abortively terminate translation. Recently, two stalled ribosome rescue systems, one involving ArfA (also known as YhdL; Chadani et al., 2010) and the other involving YaeJ (also known as ArfB; Chadani et al., 2011b; Handa et al., 2011), have been found. ArfA is a protein that requires RF2 for hydrolysis

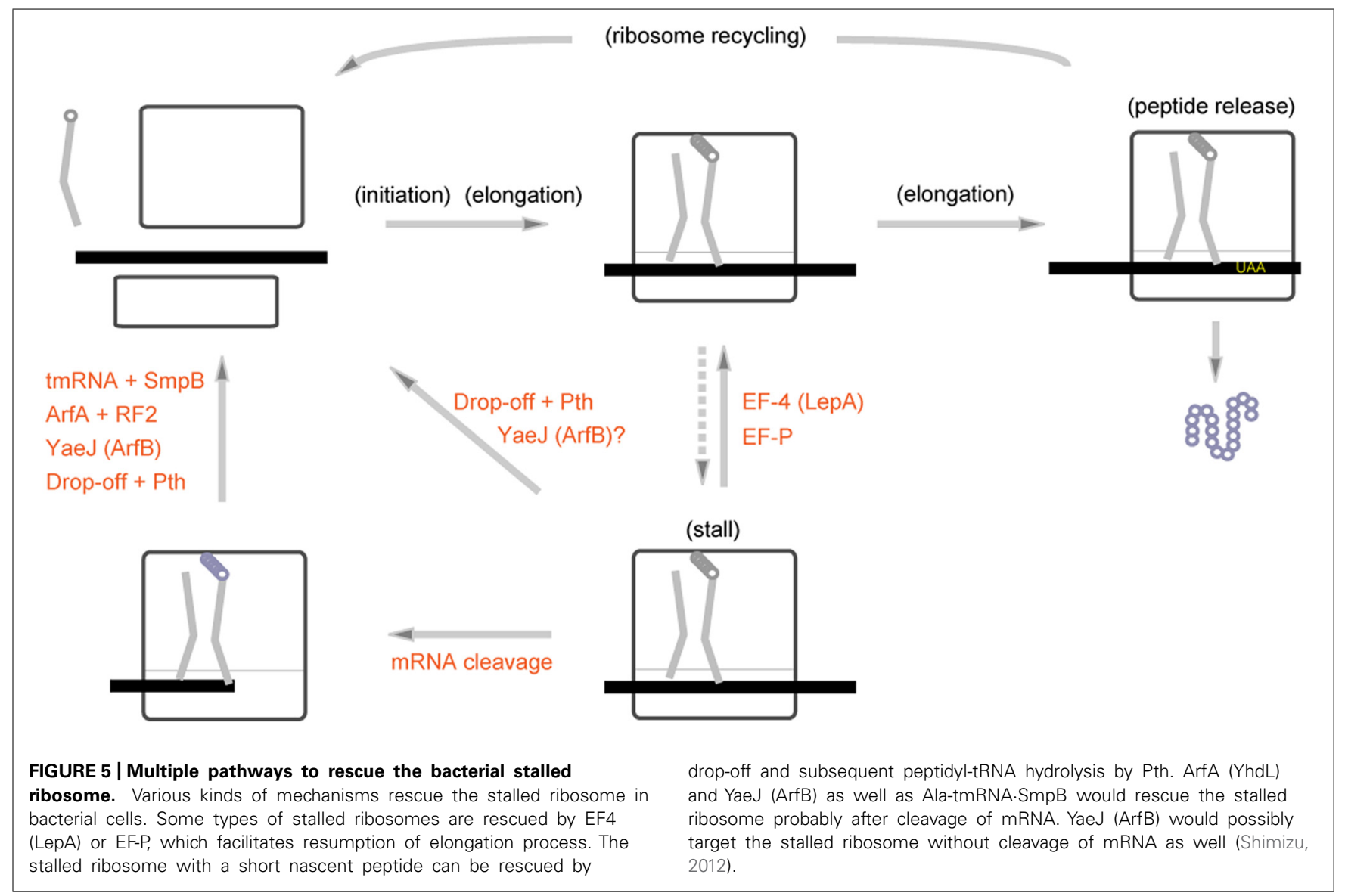


of peptidyl-tRNA (Chadani et al., 2012; Shimizu, 2012), and YaeJ is a release factor homologue but lacking a stop codon recognition capacity. These findings have encouraged us to approach the whole picture for the bacterial ribosome rescue systems in the cell. Among these ribosome rescue systems, trans-translation is the only system that involves a small RNA and is also the only system that is active in preventing truncated mRNA-derived non-functional proteins from accumulation in the cell.

Judging from its absolute occurrence among bacteria, transtranslation might be the primary ribosome rescue system in the bacterial cells. Indeed, the ArfA-mediated ribosome rescue system has been suggested to serve as the backup system for transtranslation (Chadani etal., 2011a; Garza-Sánchez et al., 2011). Since deprivation of trans-translation causes various kinds of disorders of bacterial cells and it is absent in higher eukaryotes, the trans-translation process is being used as a target for antibiotic development (Shi et al., 2011; Ramadoss et al., 2013b). A drug that specifically inhibits a step of trans-translation would make a contribution to a better understanding of the molecular mechanism of the trans-translation process.

\section{DOWNSTREAM mRNA PATH AS THE PRIMARY DETERMINANT OF THE RIBOSOME RESCUE SYSTEMS}

The C-terminal tail of YaeJ is in a situation similar to that of SmpB: it has an unstructured form in solution, although it extends to the downstream mRNA tunnel along the mRNA path with an $\alpha$-helical structure to hydrolyze the peptidyl-tRNA in the P-site of the stalled ribosome (Gagnon et al., 2012). While the trans-translation system is distributed only among bacteria with some exceptions, YaeJ or its homologue (ICT1) is present not only in bacteria but also in mitochondria (Handa et al., 2010; Richter et al., 2010). In eukaryotic cytoplasm, a complex of Dom34p (also known as Pelota) and Hbs1 (Tsuboi etal., 2012), which is structurally similar to the eRF1.eRF3 or aminoacyl-tRNA.EF-Tu complex (Chen et al., 2010), participates in recue of the stalled ribosome. The central and $\mathrm{C}$-terminal domains of $\mathrm{Hsb} 1$ have sequence and structural similarities to those of eRF3, while the N-terminal domain is distinct, which appears to recognize the downstream mRNA path of the stalled ribosome (Becker et al., 2011). Thus various types of ribosome rescue machineries would employ the interaction with the vacant downstream mRNA path to recognize the target ribosome.

\section{ACKNOWLEDGMENTS}

The authors are grateful to all those who have been involved in this work. This work was supported by Grants-in-Aid for Scientific Research (B) from the Japan Society for the Promotion of Science to Hyouta Himeno and Akira Muto and Grants-in-Aid for Young Scientist from the Japan Society for the Promotion of Science to Daisuke Kurita.

\section{REFERENCES}

Abe, T., Sakaki, K., Fujihara, A., Ujiie, H., Ushida, C., Himeno, H., et al. (2008). tmRNA-dependent trans-translation is required for sporulation in Bacillus subtilis. Mol. Microbiol. 69, 1491-1498. doi: 10.1111/j.1365-2958.2008.06381.x

Abo, T., Inada, T., Ogawa, K., and Aiba, H. (2000). SsrA-mediated tagging and proteolysis of LacI and its role in the regulation of lac operon. EMBO J. 19, 3762-3769. doi: 10.1093/emboj/19.14.3762
Akerley, B. J., Rubin, E. J., Novick, V. L., Amaya, K., Judson, N., and Mekalanos, J. J. (2002). A genome-scale analysis for identification of genes required for growth or survival of Haemophilus influenzae. Proc. Natl. Acad. Sci. U.S.A. 99, 966-971. doi: 10.1073/pnas.012602299

Asano, K., Kurita, D., Takada, K., Konno, T., Muto, A., and Himeno, H. (2005). Competition between trans-translation and termination or elongation of translation. Nucleic Acids Res. 33, 5544-5552. doi: 10.1093/nar/gki871

Barends, S., Karzai, A. W., Sauer, R. T., Wower, J., and Kraal, B. (2001). Simultaneous and functional binding of SmpB and EF-Tu-GTP to the alanyl acceptor arm of tmRNA. J. Mol. Biol. 314, 9-21. doi: 10.1006/jmbi.2001.5114

Barends, S., Wower, J., and Kraal, B. (2000). Kinetic parameters for tmRNA binding to alanyl-tRNA synthetase and elongation factor Tu from Escherichia coli. Biochemistry 39, 2652-2658. doi: 10.1021/bi992439d

Becker, T., Armache, J. P., Jarasch, A., Anger, A. M., Villa, E., Sieber, H., et al. (2011). Structure of the no-go mRNA decay complex Dom34-Hbs1 bound to a stalled 80 S ribosome. Nat. Struct. Mol. Biol. 18, 715-720. doi: 10.1038/nsmb.2057

Bessho, Y., Shibata, R., Sekine, S., Murayama, K., Higashijima, K., Hori-Takemoto, C., et al. (2007). Structural basis for functional mimicry of long-variable-arm tRNA by transfer-messenger RNA. Proc. Natl. Acad. Sci. U.S.A. 104, 8293-8298. doi: 10.1073/pnas.0700402104

Bugaeva, E. Y., Shpanchenko, O. V., Felden, B., Isaksson, L. A., and Dontsova, O. A. (2008). One SmpB molecule accompanies tmRNA during its passage through the ribosomes. FEBS Lett. 582, 1532-1536. doi: 10.1016/j.febslet.2008.03.049

Camenares, D., Dulebohn, D. P., Svetlanov, A., and Karzai, A. W. (2013). Active and accurate trans-translation requires distinct determinants in the $\mathrm{C}$-terminal tail of SmpB protein and the mRNA-like domain of transfer messenger RNA (tmRNA). J. Biol. Chem. 288, 30527-30542. doi: 10.1074/jbc.M113.503896

Chadani, Y., Ito, K., Kutsukake, K., and Abo, T. (2012). ArfA recruits release factor 2 to rescue stalled ribosomes by peptidyl-tRNA hydrolysis in Escherichia coli. Mol. Microbiol. 86, 37-50. doi: 10.1111/j.1365-2958.2012.08190.x

Chadani, Y., Matsumoto, E., Aso, H., Wada, T., Kutsukake, K., Sutou, S., et al. (2011a). trans-translation-mediated tight regulation of the expression of the alternative ribosome-rescue factor ArfA in Escherichia coli. Genes Genet. Syst. 86, 151-163. doi: 10.1266/ggs.86.151

Chadani, Y., Ono, K., Kutsukake, K., and Abo, T. (2011b). Escherichia coli YaeJ protein mediates a novel ribosome-rescue pathway distinct from SsrA- and ArfA-mediated pathways. Mol. Microbiol. 80, 772-785. doi: 10.1111/j.13652958.2011.07607.x

Chadani, Y., Ono, K., Ozawa, S., Takahashi, Y., Takai, K., Nanamiya, H., et al. (2010). Ribosome rescue by Escherichia coli ArfA (YhdL) in the absence of trans-translation system. Mol. Microbiol. 80, 772-775. doi: 10.1111/j.13652958.2011.07607.x

Chen, L., Muhlrad, D., Hauryliuk, V., Cheng, Z., Lim, M. K., Shyp, V., et al. (2010). Structure of the Dom34-Hbs1 complex and implications for no-go decay. Nat. Struct. Mol. Biol. 17, 1233-1240. doi: 10.1038/nsmb.1922

Cheng, K., Ivanova, N., Scheres, S. H., Pavlov, M. Y., Carazo, J. M., Hebert, H., et al. (2010). tmRNA.SmpB complex mimics native aminoacyl-tRNAs in the A site of stalled ribosomes. J. Struct. Biol. 169, 342-348. doi: 10.1016/j.jsb.2009. 10.015

Choy, J. S., Aung, L. L., and Karzai, A. W. (2007). Lon protease degrades transfer-messenger RNA-tagged proteins. J. Bacteriol. 189, 6564-6571. doi: 10.1128/JB.00860-07

Collier, J., Binet, E., and Bouloc, P. (2002). Competition between SsrA tagging and translational termination at weak stop codons in Escherichia coli. Mol. Microbiol. 45, 745-754. doi: 10.1046/j.1365-2958.2002.03045.x

Doerfel, L. K., Wohlgemuth, I., Kothe, C., Peske, F., Urlaub, H., and Rodnina, M. V. (2013). EF-P is essential for rapid synthesis of proteins containing consecutive proline residues. Science 339, 85-88. doi: 10.1126/science. 1229017

Dong, G., Nowakowski, J., and Hoffman, D. W. (2002). Structure of small protein B: the protein component of the tmRNA-SmpB system for ribosome rescue. EMBO J. 21, 1845-1854. doi: 10.1093/emboj/21.7.1845

Felden, B., Hanawa, K., Atkins, J. F., Himeno, H., Muto, A., Gesteland, R. F., et al. (1998). Presence and location of modified nucleotides in E. coli tmRNA. Structural mimicry with tRNA acceptor branches. EMBO J. 17, 3188-3196. doi: 10.1093/emboj/17.11.3188

Felden, B., and Gillet, R. (2011). SmpB as the handyman of tmRNA during transtranslation. RNA Biol. 8, 440-449. doi: 10.4161/rna.8.3.15387 
Felden, B., Himeno, H., Muto, A., McCutcheon, J. P., Atkins, J. F., and Gesteland, R. F. (1997). Probing the structure of the Escherichia coli 10Sa RNA (tmRNA). RNA 3, 89-103.

Feng, S., Chen, Y., Kamada, K., Wang, H., Tang, K., Wang, M., et al. (2013). YoeBribosome structure: a canonical RNase that requires the ribosome for its specific activity. Nucleic Acids Res. 41, 9549-9556. doi: 10.1093/nar/gkt742

Flynn, J. M., Levchenko, I., Seidel, M., Wickner, S. H., Sauer, R. T., and Baker, T. A. (2001). Overlapping recognition determinants within the ssrA degradation tag allow modulation of proteolysis. Proc. Natl. Acad. Sci. U.S.A. 98, 10584-10589. doi: 10.1073/pnas. 191375298

Frank, J., and Agrawal, R. K. (2000). A ratchet-like inter-subunit reorganization of the ribosome during translocation. Nature 406, 318-322. doi: 10.1038/35018597

Fu, J., Hashem, Y., Wower, I., Lei, J., Liao, H. Y., Zwieb, C., et al. (2010). Visualizing the transfer-messenger RNA as the ribosome resumes translation. EMBO J. 29, 3819-3825. doi: 10.1038/emboj.2010.255

Fujihara, A., Tomatsu, H., Inagaki, S., Tadaki, T., Ushida, C., Himeno, H., et al. (2002). Detection of tmRNA-mediated trans-translation products in Bacillus subtilis. Genes Cells 7, 343-350. doi: 10.1046/j.1365-2443.2002.00523.x

Gagnon, M. G., Seetharaman, S. V., Bulkley, D., and Steitz, T. A. (2012). Structura basis for the rescue of stalled ribosomes: structure of YaeJ bound to the ribosome. Science 335, 1370-1372. doi: 10.1126/science. 1217443

Garza-Sánchez, F., Schaub, R. E., Janssen, B. D., and Hayes, C. S. (2011). tmRNA regulates synthesis of the ArfA ribosome rescue factor. Mol. Microbiol. 80, 12041219. doi: 10.1111/j.1365-2958.2011.07638.x

Garza-Sánchez, F., Shoji, S., Fredrick, K., and Hayes, C. S. (2009). RNase II is important for A-site mRNA cleavage during ribosome pausing. Mol. Microbiol. 73, 882-897. doi: 10.1111/j.1365-2958.2009.06813.x

Gillet, R., Kaur, S., Li, W., Hallier, M., Felden, B., and Frank, J. (2006) Scaffolding as an organizing principle in trans-translation: the roles of small protein B and ribosomal protein S1. J. Biol. Chem. 282, 6356-6363. doi: 10.1074/jbc.M609658200

Gong, M., Cruz-Vera, L. R., and Yanofsky, C. (2007). Ribosome recycling factor and release factor 3 action promotes TnaC-Peptidyl-tRNA dropoff and relieves ribosome stalling during tryptophan induction of tha operon expression in Escherichia coli. J. Bacteriol. 189, 3147-3155. doi: 10.1128/JB.01868-06

Gottesman, S., Roche, E., Zhou, Y., and Sauer, R. T. (1998). The ClpXP and ClpAP proteases degrade proteins with carboxy-terminal peptide tails added by the SsrAtagging system. Genes Dev. 12, 1338-1347. doi: 10.1101/gad.12.9.1338

Gueneau de Novoa, P., and Williams, K. P. (2004). The tmRNA website: reductive evolution of tmRNA in plastids and other endosymbionts. Nucleic Acids Res. 32, D104-D108. doi: 10.1093/nar/gkh102

Gutmann, S., Haebel, P. W., Metzinger, L., Sutter, M., Felden, B., and Ban, N. (2003). Crystal structure of the transfer-RNA domain of transfer-messenger RNA in complex with SmpB. Nature 424, 5503-5509. doi: 10.1038/nature01831

Hanawa-Suetsugu, K., Bordeau, V., Himeno, H., Muto, A., and Felden, B. (2001) Importance of the conserved nucleotides around the tRNA-like structure of Escherichia coli transfer-messenger RNA for protein tagging. Nucleic Acids Res. 29, 4663-4673. doi: 10.1093/nar/29.22.4663

Hanawa-Suetsugu, K., Takagi, M., Inokuchi, H., Himeno, H., and Muto, A. (2002). SmpB functions in various steps of trans-translation. Nucleic Acids Res. 30, 1620 1629. doi: 10.1093/nar/30.7.1620

Handa, Y., Hikawa, Y., Tochio, N., Kogure, H., Inoue, M., Koshiba, S., et al. (2010). Solution structure of the catalytic domain of the mitochondrial protein ICT1 that is essential for cell vitality. J. Mol. Biol. 404, 260-273. doi 10.1016/j.jmb.2010.09.033

Handa, Y., Inaho, N., and Nameki, N. (2011). YaeJ is a novel ribosome-associated protein in Escherichia coli that can hydrolyze peptidyl-tRNA on stalled ribosomes. Nucleic Acids Res. 39, 1739-1748. doi: 10.1093/nar/gkq1097

Herman, C., Thévenet, D., Bouloc, P., Walker, G. C., and D’Ari, R. (1998). Degradation of carboxy-terminal-tagged cytoplasmic proteins by the Escherichia col protease HflB (FtsH). Genes Dev. 12, 1348-1355. doi: 10.1101/gad.12.9.1348

Heurgué-Hamard, V., Karimi, R., Mora, L., MacDougall, J., Leboeuf, C., Grentzmann, G., et al. (1998). Ribosome release factor RF4 and termination factor RF3 are involved in dissociation of peptidyl-tRNA from the ribosome. EMBO J. 17, 808-816. doi: 10.1093/emboj/17.3.808

Himeno, H., Sato, M., Tadaki, T., Fukushima, M., Ushida, C., and Muto, A. (1997). In vitro trans translation mediated by alanine-charged 10Sa RNA. J. Mol. Biol. 268, 803-808. doi: 10.1006/jmbi.1997.1011
Huang, C., Wolfgang, M. C., Withey, J., Koomey, M., and Friedman, D. I. (2000) Charged tmRNA but not tmRNA-mediated proteolysis is essential for Neisseria gonorrhoeae viability. EMBO J. 19, 1098-1107. doi: 10.1093/emboj/19.5.1098

Hutchison, C. A., Peterson, S. N., Gill, S. R., Cline, R. T., White, O., Fraser, C. M., et al. (1999). Global transposon mutagenesis and a minimal mycoplasma genome. Science 286, 2165-2169. doi: 10.1126/science.286.5447.2165

Ito, K., Tadaki, T., Lee, S., Takada, K., Muto, A., and Himeno, H. (2002). Transtranslation mediated by Bacillus subtilis tmRNA. FEBS Lett. 516, 245-252. doi: 10.1016/S0014-5793(02)02561-9

Ivanova, N., Pavlov, M. Y., and Ehrenberg, M. (2005). tmRNA-induced release of messenger RNA from stalled ribosomes. J. Mol. Biol. 350, 897-905. doi: 10.1016/j.jmb.2005.05.033

Ivanova, N., Pavlov, M. Y., Felden, B., and Ehrenberg, M. (2004). Ribosome rescue by tmRNA requires truncated mRNAs. J. Mol. Biol. 338, 33-41. doi: 10.1016/j.jmb.2004.02.043

Jacob, Y., Seif, E., Paquet, P.-O., and Lang, B. F. (2004). Loss of the mRNAlike region in mitochondrial tmRNAs of jakobids. RNA 10, 605-614. doi: 10.1261/rna.5227904

Jacob, Y., Sharkady, S. M., Bhardwaj, K., Sanda, A. A., and Williams, K. P. (2005). Function of the $S m p B$ tail in transfer-messenger RNA translation revealed by a nucleus-encoded form. J. Biol. Chem. 280, 5503-5509. doi: 10.1074/jbc.M409277200

Karzai, A. W., Susskind, M. M., and Sauer, R. T. (1999). SmpB, a unique RNA-binding protein essential for the peptide-tagging activity of SsrA (tmRNA). EMBO J. 18, 3793-3799. doi: 10.1093/emboj/18.13.3793

Kaur, S., Gillet, R., Li, W., Gursky, R., and Frank, J. (2006). Cryo-EM visualization of transfer messenger RNA with two SmpBs in a stalled ribosome. Proc. Natl. Acad. Sci. U.S.A. 103, 16484-16489. doi: 10.1073/pnas.0607438103

Keiler, K. C. (2008). Biology of trans-translation. Annu. Rev. Microbiol. 62, 133-151. doi: 10.1146/annurev.micro.62.081307.162948

Keiler, K. C., and Shapiro, L. (2003a). tmRNA in Caulobacter crescentus is cell cycle regulated by temporally controlled transcription and RNA degradation. $J$. Bacteriol. 185, 1825-1830. doi: 10.1128/JB.185.6.1825-1830.2003

Keiler, K. C., and Shapiro, L. (2003b). tmRNA is required for correct timing of DNA replication in Caulobacter crescentus. J. Bacteriol. 185, 573-580. doi: 10.1128/JB.185.2.573-580.2003

Keiler, K. C., Waller, P. R., and Sauer, R. T. (1996). Role of a peptide tagging system in degradation of proteins synthesized from damaged messenger RNA. Science 271, 990-993. doi: 10.1126/science.271.5251.990

Komine, Y., Kitabatake, M., Yokogawa, T., Nishikawa, K., and Inokuchi, H. (1994). A tRNA-like structure is present in 10Sa RNA, a small stable RNA from Escherichia coli. Proc. Natl. Acad. Sci. U.S.A. 91, 9223-9227. doi: 10.1073/pnas.91. 20.9223

Konno, T., Kurita, D., Takada, K., Muto, A., and Himeno, H. (2007). A functional interaction of SmpB with tmRNA for determination of the resuming point of trans-translation. RNA 13, 1723-1731. doi: 10.1261/rna.604907

Konno, T., Takahashi, T., Kurita, D., Muto, A., and Himeno, H. (2004). A minimum structure of aminoglycosides that causes an initiation shift of trans-translation. Nucleic Acids Res. 32, 4119-4126. doi: 10.1093/nar/gkh750

Kurita, D., Muto, A., and Himeno, H. (2010). Role of the C-terminal tail of SmpB in the early stage of trans-translation. RNA 16, 980-990. doi: 10.1261/rna. 1916610

Kurita, D., Muto, A., and Himeno, H. (2011). tRNA/mRNA mimicry by tmRNA and SmpB in trans-translation. J. Nucleic Acids 2011, Article ID 130581. doi: 10.4061/2011/130581

Kurita, D., Muto, A., and Himeno, H. (2012). In vitro trans-translation assay. Methods Mol. Biol. 905, 311-325. doi: 10.1007/978-1-61779-949-5_20

Kurita, D., Sasaki, R., Muto, A., and Himeno, H. (2007). Interaction of SmpB with ribosome from directed hydroxyl radical probing. Nucleic Acids Res. 35, 7248-7255. doi: 10.1093/nar/gkm677

Kuroha, K., Horiguchi, N., Aiba, H., and Inada, T. (2009). Analysis of nonstop mRNA translation in the absence of tmRNA in Escherichia coli. Genes Cells 14, 739-749. doi: 10.1111/j.1365-2443.2009.01304.x

Lee, S., Ishii, M., Tadaki, T., Muto, A., and Himeno, H. (2001). Determinants on tmRNA for initiating efficient and precise trans-translation: some mutations upstream of the tag-encoding sequence of Escherichia coli tmRNA shift the initiation point of trans-translation in vitro. RNA 7, 999-1012. doi: $10.1017 /$ S1355838201010342 
Miller, M. R., and Buskirk, A. R. (2014). An unusual mechanism for EF-Tu activation during tmRNA-mediated ribosome rescue. RNA 20, 228-235. doi: 10.1261/rna.042226.113

Miller, M. R., Liu, Z., Cazier, D. J., Gebhard, G. M., Herron, S. R., Zaher, H. S., et al. (2011). The role of SmpB and the ribosomal decoding center in licensing tmRNA entry into stalled ribosomes. RNA 17, 1727-1736. doi: 10.1261/rna.2821711

Muto, A., Fujihara, A., Ito, K., Matsuno, J., Ushida, C., and Himeno, H. (2000). Requirement of transfer-messenger RNA (tmRNA) for the growth of Bacillus subtilis under stresses. Genes Cells 5, 627-636. doi: 10.1046/j.1365-2443.2000.00356.x

Muto, A., Sato, M., Tadaki, T., Fukushima, M., Ushida, C., and Himeno, H. (1996). Structure and function of bacterial 10Sa RNA: trans-translation system. Biochimie 78, 985-991. doi: 10.1016/S0300-9084(97)86721-1

Muto, A., Ushida, C., and Himeno, H. (1998). A bacterial RNA that functions as both a tRNA and an mRNA. Trends Biochem. Sci. 23, 25-29. doi: 10.1016/S09680004(97)01159-6

Nameki, N., Tadaki, T., Muto, A., and Himeno, H. (1999a). Amino acid acceptor identity switch of Escherichia coli tmRNA from alanine to histidine in vitro. $J$. Mol. Biol. 289, 1-7. doi: 10.1006/jmbi.1999.2754

Nameki, N., Felden, B., Atkins, J. F., Gesteland, R. F., Himeno, H., and Muto, A. (1999b). Functional and structural analysis of a pseudoknot upstream of the tag-encoded sequence in E. coli tmRNA. J. Mol. Biol. 286, 733-744. doi: 10.1006/jmbi.1998.2487

Nameki, N., Someya, T., Okano, S., Suemasa, R., Kimoto, M., Hanawa-Suetsugu, K., et al. (2005). Interaction analysis between tmRNA and SmpB from Thermus thermophilus. J. Biochem. 138, 729-739. doi: 10.1093/jb/mvi180

Neubauer, C., Gillet, R., Kelley, A. C., and Ramakrishnan, V. (2012). Decoding in the absence of a codon by tmRNA and SmpB in the ribosome. Science 355, 1366-1369. doi: 10.1126/science.1217039

Nissen, P., Kjeldgaard, M., Thirup, S., Polekhina, G., Reshetnikova, L., Clark, B. F. C., et al. (1995). Crystal structure of the ternary complex of Phe-tRNAPhe, EF-Tu, and a GTP analog. Science 270, 1464-1472. doi: 10.1126/science.270.52 41.1464

Nonin-Lecomte, S., Germain-Amiot, N., Gillet, R., Hallier, M., Ponchon, L., Dardel, F., et al. (2009). Ribosome hijacking: a role for small protein B during transtranslation. EMBO Rep. 10, 160-165. doi: 10.1038/embor.2008.243

Pech, M., Karim, Z., Yamamoto, H., Kitakawa, M., Qin, Y., and Nierhaus, K. H. (2011). Elongation factor 4 (EF4/LepA) accelerates protein synthesis at increased Mg2+ concentrations. Proc. Natl. Acad. Sci. U.S.A. 108, 3199-3203. doi: $10.1073 /$ pnas. 1012994108

Pedersen, K., Zavialov, A. V., Pavlov, M. Y., Elf, J., Gerdes, K., and Ehrenberg, M. (2003). The bacterial toxin RelE displays codon-specific cleavage of mRNAs in the ribosomal A site. Cell 112, 131-140. doi: 10.1016/S0092-8674(02) 01248-5

Qi, H., Shimizu, Y., and Ueda, T. (2007). Ribosomal protein S1 is not essential for the trans-translation machinery. J. Mol. Biol. 368, 845-852. doi: 10.1016/j.jmb.2007.02.068

Ramadoss, N. S., Zhou, X., and Keiler, K. C. (2013a). tmRNA is essential in Shigella flexneri. PLoS ONE 8:e57537. doi: 10.1371/journal.pone.0057537

Ramadoss, N. S., Alumasa, J. N., Cheng, L., Wang, Y., Li, S., Chambers, B. S., et al. (2013b). Small molecule inhibitors of trans-translation have broadspectrum antibiotic activity. Proc. Natl. Acad. Sci. U.S.A. 110, 10282-10287. doi: $10.1073 /$ pnas. 1302816110

Ramrath, D. J., Yamamoto, H., Rother, K., Wittek, D., Pech, M., Mielke, T., et al. (2012). The complex of tmRNA-SmpB and EF-G on translocating ribosomes. Nature 485, 526-529. doi: 10.1038/nature11006

Ranquet, C., and Gottesman, S. (2007). Translational regulation of the Escherichia coli stress factor RpoS: a role for SsrA and Lon. J. Bacteriol. 189, 4872-4879. doi: 10.1128/JB.01838-06

Richter, R., Rorbach, J., Pajak, A., Smith, P. M., Wessels, H. J., Huynen, M. A., et al. (2010). A functional peptidyl-tRNA hydrolase, ICT1, has been recruited into the human mitochondrial ribosome. EMBO J. 29, 1116-1125. doi: 10.1038 /emboj.2010.14

Roche, E. D., and Sauer, R. T. (2001). Identification of endogenous SsrA-tagged proteins reveals tagging at positions corresponding to stop codons. J. Biol. Chem. 276, 28509-28515. doi: 10.1074/jbc.M103864200

Rudinger-Thirion, J., Giegé, R., and Felden, B. (1999). Aminoacylated tmRNA from Escherichia coli interacts with prokaryotic elongation factor Tu. RNA 5, 989-992. doi: 10.1017/S135583829999101X
Saguy, M., Gillet, R., Skorski, P., Hermann-Le Denmat, S., and Felden, B. (2007). Ribosomal protein S1 influences trans-translation in vitro and in vivo. Nucleic Acids Res. 35, 2368-2376. doi: 10.1093/nar/gkm100

Shi, W., Zhang, X., Jiang, X., Yuan, H., Lee, J. S., Barry, C. E. III, et al. (2011). Pyrazinamide inhibits trans-translation in Mycobacterium tuberculosis. Science 333, 1630-1632. doi: 10.1126/science. 1208813

Shimizu, Y. (2012). ArfA recruits RF2 into stalled ribosomes. J. Mol. Biol. 423, 624-631. doi: 10.1016/j.jmb.2012.08.007

Shimizu, Y., and Ueda, T. (2002). The role of SmpB protein in trans-translation. FEBS Lett. 514, 74-77. doi: 10.1016/S0014-5793(02)02333-5

Shimizu, Y., and Ueda, T. (2006). SmpB triggers GTP hydrolysis of elongation factor Tu on ribosomes by compensating for the lack of codon-anticodon interaction during trans-translation initiation. J. Biol. Chem. 281, 15987-15996. doi: 10.1074/jbc.M512165200

Shin, J.-H., and Price, C. W. (2007). The SsrA-SmpB ribosome rescue system is important for growth of Bacillus subtilis at low and high temperature. J. Bacteriol. 189, 3729-3737. doi: 10.1128/JB.00062-07

Singh, N. S., Ahmad, R., Sangeetha, R., and Varshney, U. (2008). Recycling of ribosomal complexes stalled at the step of elongation in Escherichia coli. J. Mol. Biol. 380, 451-464. doi: 10.1016/j.jmb.2008.05.033

Singh, N. S., and Varshney, U. (2004). A physiological connection between tmRNA and peptidyl-tRNA hydrolase functions in Escherichia coli. Nucleic Acids Res. 32, 6028-6037. doi: 10.1093/nar/gkh924

Someya, T., Nameki, N., Hosoi, H., Suzuki, S., Hatanaka, H., Fujii, M., et al. (2003). Solution structure of a tmRNA-binding protein, $\mathrm{SmpB}$, from Thermus thermophilus. FEBS Lett. 535, 94-100. doi: 10.1016/S0014-5793(02)03880-2

Sundermeier, T. R., Dulebohn, D. P., Cho, H. J., and Karzai, A. W. (2005). A previously uncharacterized role for small protein $B(\mathrm{SmpB})$ in transfer messenger RNA-mediated trans-translation. Proc. Natl. Acad. Sci. U.S.A. 102, 2316-2321. doi: 10.1073/pnas.0409694102

Tadaki, T., Fukushima, M., Ushida, C., Himeno, H., and Muto, A. (1996). Interaction of 10Sa RNA with ribosomes in Escherichia coli. FEBS Lett. 399, 223-226. doi: 10.1016/S0014-5793(96)01330-0

Takada, K., Takemoto, C., Kawazoe, M., Konno, T., Hanawa-Suetsugu, K., Lee, S., et al. (2007). In vitro trans-translation of Thermus thermophilus: ribosomal protein $\mathrm{S} 1$ is not required for the early stage of trans-translation. RNA 13, 503510. doi: 10.1261/rna.363207

Takahashi, T., Konno, T., Muto, A., and Himeno, H. (2003). Various effects of paromomycin on tmRNA-directed trans-translation. J. Biol. Chem. 278, 2767227680. doi: 10.1074/jbc.M211724200

Tsuboi, T., Kuroha, K., Kudo, K., Makino, S., Inoue, E., Kashima, I., et al. (2012). Dom34:hbs1 plays a general role in quality-control systems by dissociation of a stalled ribosome at the $3^{\prime}$ end of aberrant mRNA. Mol. Cell 46, 518-529. doi: 10.1016/j.molcel.2012.03.013

Tu, G. F., Reid, G. E., Zhang, J. G., Moritz, R. L., and Simpson, R. J. (1995). C-terminal extension of truncated recombinant proteins in Escherichia coli with a 10Sa RNA decapeptide. J. Biol. Chem. 270, 9322-9326. doi: 10.1074/jbc.270.16.9322

Ude, S., Lassak, J., Starosta, A. L., Kraxenberger, T., Wilson, D. N., and Jung, K. (2013). Translation elongation factor EF-P alleviates ribosome stalling at polyproline stretches. Science 339, 82-85. doi: 10.1126/science.1228985

Ujiie, H., Matsutani, T., Tomatsu, H., Fujihara, A., Ushida, C., Miwa, Y., et al. (2009). Trans-translation is involved in the CcpA-dependent tagging and degradation of TreP in Bacillus subtilis. J. Biochem. 145, 59-66. doi: 10.1093/jb/mvn 143

Ushida, C., Himeno, H., Watanabe, T., and Muto, A. (1994). tRNA-like structures in 10Sa RNAs of Mycoplasma capricolum and Bacillus subtilis. Nucleic Acids Res. 22, 3392-3396. doi: 10.1093/nar/22.16.3392

Watts, T., Cazier, D., Healey, D., and Buskirk, A. (2009). SmpB contributes to reading frame selection in the translation of transfer-messenger RNA. J. Mol. Biol. 391, 275-281. doi: 10.1016/j.jmb.2009.06.037

Weis, F., Bron, P., Rolland, J. P., Thomas, D., Felden, B., and Gillet, R. (2010a). Accommodation of tmRNA-SmpB into stalled ribosomes: a cryo-EM study. RNA 16, 299-306. doi: 10.1261/rna.1757410

Weis, F., Bron, P., Giudice, E., Rolland, J. P., Thomas, D., Felden, B., et al. (2010b). tmRNA-SmpB: a journey to the centre of the bacterial ribosome. EMBO J. 29, 3810-3818. doi: 10.1038/emboj.2010.252

Williams, K. P., Martindale, K. A., and Bartel, D. P. (1999). Resuming translation on tmRNA: a unique mode of determining a reading frame. EMBO J. 18, 5423-5433. doi: 10.1093/emboj/18.19.5423 
Wower, I. K., Zwieb, C. W., Guven, S. A., and Wower, J. (2000). Binding and cross-linking of tmRNA to ribosomal protein S1, on and off the Escherichia coli ribosome. EMBO J. 19, 6612-6621. doi: 10.1093/emboj/19. 23.6612

Yamamoto, Y., Sunohara, T., Jojima, K., Inada, T., and Aiba, H. (2003). SsrA-mediated trans-translation plays a role in mRNA quality control by facilitating degradation of truncated mRNAs. RNA 9, 408-418. doi: 10.1261/rna.21 74803

Conflict of Interest Statement: The authors declare that the research was conducted in the absence of any commercial or financial relationships that could be construed as a potential conflict of interest.
Received: 15 December 2013; accepted: 04 February 2014; published online: 20 February 2014

Citation: Himeno H, Kurita D and Muto A (2014) Mechanism of trans-translation revealed by in vitro studies. Front. Microbiol. 5:65. doi: 10.3389/fmicb.2014.00065

This article was submitted to Microbial Physiology and Metabolism, a section of the journal Frontiers in Microbiology.

Copyright (C) 2014 Himeno, Kurita and Muto. This is an open-access article distributed under the terms of the Creative Commons Attribution License (CC BY). The use, distribution or reproduction in other forums is permitted, provided the original author(s) or licensor are credited and that the original publication in this journal is cited, in accordance with accepted academic practice. No use, distribution or reproduction is permitted which does not comply with these terms. 